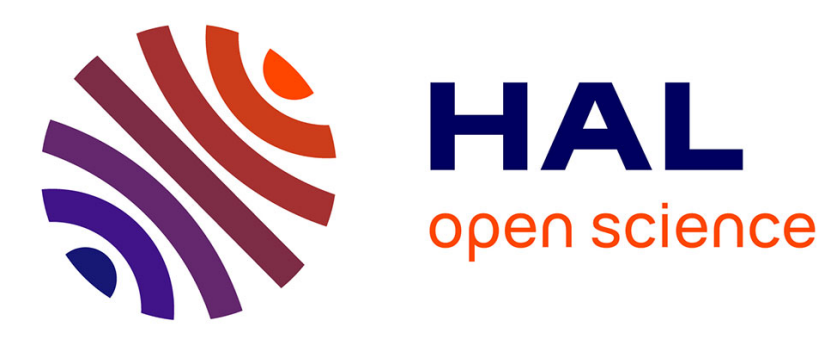

\title{
La vie à domicile des personnes handicapées ou dépendantes : du besoin d'aide aux arrangements pratiques \\ Jean-Sébastien Eideliman, Séverine Gojard
}

\section{To cite this version:}

Jean-Sébastien Eideliman, Séverine Gojard. La vie à domicile des personnes handicapées ou dépendantes: du besoin d'aide aux arrangements pratiques. Retraite et société, 2008, 1 (53), pp.89-111. hal-00351420

\section{HAL Id: hal-00351420 \\ https://hal.science/hal-00351420}

Submitted on 9 Feb 2009

HAL is a multi-disciplinary open access archive for the deposit and dissemination of scientific research documents, whether they are published or not. The documents may come from teaching and research institutions in France or abroad, or from public or private research centers.
L'archive ouverte pluridisciplinaire HAL, est destinée au dépôt et à la diffusion de documents scientifiques de niveau recherche, publiés ou non, émanant des établissements d'enseignement et de recherche français ou étrangers, des laboratoires publics ou privés. 
La vie à domicile

des personnes

handicapées

ou dépendantes:

du besoin d'aide

aux arrangements

pratiques 
L'accroissement du poids des plus âgés au sein de la population française, ainsi que d'autres événements conjoncturels comme la canicule de l'été 2003, ont porté depuis une quinzaine d'années le problème des «personnes âgées dépendantes» en bonne place sur I'agenda politique, tout comme sur la scène médiatique ${ }^{1}$ (Frinault, 2005). La prise en charge des personnes handicapées, quoique moins présente dans le débat public, a également fait l'objet d'une loi importante en 2005 («année du handicap» en France) portant sur «l'égalité des droits et des chances, la participation et la citoyenneté des personnes handicapées ${ }^{2}{ }^{2}$, remplaçant la loi d'orientation de $1975^{3}$. Notons que ces deux champs, qui peuvent tous deux renvoyer à des logiques de caring, sont rarement pensés conjointement (Frinault, 2005), bien que le thème des personnes handicapées vieillissantes soit en passe de devenir un sujet d'étude à part entière (Azéma, Martinez, 2005).

Ils évoluent cependant de façon convergente, la prise en charge à domicile se développant au détriment de I'institutionnalisation. Ces évolutions se traduisent sur le plan juridique: la loi d'orientation de 1975 sur le handicap prônait clairement le maintien en milieu ordinaire aussi souvent que possible et a été suivie de nombreux décrets visant à l'amélioration (lente dans les faits) tant de l'intégration scolaire que de l'insertion professionnelle des personnes handicapées (Didier-Courbin, Gilbert, 2005). Plus récemment, la nouvelle loi de 2005 a conforté les dispositifs visant à faciliter le maintien à domicile. La prise en charge des personnes âgées dépendantes n'échappe pas à ce mouvement, qu'elle épouse plus tardivement, à partir des années quatre-vingt-dix (Joël, 2002). Les mises en place successives de la Prestation spécifique dépendance (PSD) - en 1997 - puis de I'Allocation personnalisée d'autonomie (Apa) - en 2002 - (Kerjosse, 2002 ; Espagnol, 2007) s'inscrivent dans ce même mouvement de promotion du maintien

1 Nous remercions vivement l'ensemble de l'équipe Medips (Modélisation de l'économie domestique et incidence des politiques sociales), qui nous a soutenus à toutes les étapes de la production de cet article, et en particulier Aude Béliard, Émilie Biland, Agnès Gramain, Delphine Roy et Florence Weber pour leurs relectures et suggestions. Nous tenons également à exprimer tous nos remerciements à Pierre Strobel pour ses commentaires constructifs sur une précédente version de ce texte.

2 Loi 2005-102 du 11 février 2005.

3 Loi 1975-534 du 30 juin 1975. 
à domicile, qui rencontre les aspirations des personnes âgées dépendantes elles-mêmes et de leur entourage ${ }^{4}$. Dans un contexte démographique rendant difficile I'accueil en institution d'un nombre croissant de personnes, ces mesures correspondent également à la volonté politique de lutter contre le chômage en créant davantage d'emplois dits de proximité, comme les aides à domicile (Thomas, 2005), et en s'appuyant sur les «solidarités familiales», redécouvertes à I'occasion (Martin, 2002).

Le recours accru à I'entourage pour assurer le maintien à domicile suppose une mobilisation dont les conditions sociales et matérielles ne sont pas toujours réunies et qui nécessite dans tous les cas une réorganisation des contributions domestiques des uns et des autres (Weber et al., 2003). Nous proposons dans cet article d'analyser des arrangements pratiques et d'éclairer ainsi I'ensemble des modalités concrètes d'organisation de la vie quotidienne qui permettent de surmonter des problèmes de santé handicapants. Ces arrangements peuvent s'inscrire dans trois sphères: au sein du ménage, ils concernent la prise en charge des différentes tâches domestiques ${ }^{5}$; au sein de la parenté, ils recouvrent des transferts de ressources (en temps, en argent, en compétences) non nécessairement formalisées dans un cadre contractuel $^{6}$; plus largement encore, nous considérons qu'ils peuvent inclure le recours à des aidants non familiaux, professionnels ou non.

Pour conduire notre étude, nous nous appuyons sur une exploitation secondaire de I'enquête Handicaps-Incapacités-Dépendance ${ }^{7}$ menée en 1999 par I'Insee auprès de personnes à domicile. Cette enquête présente le grand avantage de ne pas présupposer une définition particulière du handicap ou de la dépendance (Mormiche, 2003). Parmi la population enquêtée par I'Insee ${ }^{8}$, nous avons conservé exclusivement les personnes rencontrant quotidiennement des difficultés importantes ${ }^{9}$.

4 Ces aspirations peuvent être d'une façon générale reliées à la diffusion, dans la deuxième moitié du $\mathrm{xx}^{\mathrm{e}}$ siècle, de la norme d'autonomie dans les sociétés occidentales modernes (Ehrenberg, 1998).

5 Au sens le plus usuel, cf. par exemple Bauer, 2007.

$6 \mathrm{Au}$ sens des «arrangements privés» (Pennec, 2005) ou encore des "arrangements de famille» (Bessière, 2004).

7 Cette exploitation a été menée dans le cadre de l'appel d'offre MiRe-Drees-Inserm, 2002 «Analyses secondaires de l'enquête HID» (Biland et al., 2004).

8 Un questionnaire court, appelé "Vie quotidienne et santé», a été renseigné par environ 400000 personnes en même temps que le recensement de la population française de 1999 et a permis de construire l'échantillon de l'enquête HID en surreprésentant les personnes rencontrant des difficultés quotidiennes (Ravaud et al., 2002).

9 La question du maintien à domicile se pose en effet de manière moins importante pour les personnes qui ne déclarent que des difficultés légères, voire pas de difficultés du tout. Nous avons ainsi retenu les deux tiers environ de la population initiale, soit très exactement 10000 individus. On trouvera en annexe la distribution des principales variables utilisées pour décrire cette population. 
Retraite et Société / Nº 53 Janvier 2008

Le fait que nous ne travaillons que sur des individus vivant en domicile ordinaire, et non en institution, rend notre échantillon très spécifique. En effet, les réseaux familiaux et les positions sociales des personnes ont des répercussions importantes aussi bien sur la capacité à faire face aux limitations fonctionnelles (Cambois, Robine, 2003b) que sur les transitions entre domicile et institution (Désesquelles, Brouard, 2003; Mormiche, Boissonnat, 2003). Selon que I'on est un homme ou une femme, que I'on appartient à tel ou tel milieu social, à telle ou telle classe d'âges, les conditions sociales du maintien à domicile des personnes qui rencontrent les «mêmes » difficultés quotidiennes diffèrent fortement. Cet effet de sélection est sous-jacent à toutes les analyses qui vont suivre.

Celles-ci visent à réexaminer, à travers la description concrète de I'organisation quotidienne des personnes observées, les notions d'aide, de besoin d'aide et d'aidants, en insistant sur l'ensemble des arrangements pratiques mis en place pour faire face aux difficultés de la vie quotidienne à domicile. Nous n'adoptons donc pas une logique de description des populations dépendantes ou handicapées ${ }^{10}$ mais nous cherchons, au sein de ces populations, à mettre en évidence des corrélations entre variables révélatrices de logiques d'organisation quotidienne. La mise en évidence des principales formes d'arrangements pratiques suppose au préalable une mise en question des notions de besoin d'aide puis d'aidants.

\section{- Vivre avec ou sans aide}

La définition même des personnes handicapées ou dépendantes suppose que celles-ci rencontrent des difficultés plus ou moins importantes pour effectuer certaines des activités de la vie quotidienne. Lorsque I'on s'intéresse à la manière dont ces personnes s'organisent, on s'attend donc à observer une présence massive d'aidants, faute de quoi l'on est tenté de conclure à un besoin d'aide non comblé. Mais comment mesurer ce besoin? Et dans quelle mesure rend-il compte du point de vue des personnes dépendantes elles-mêmes?

\section{À l'épreuve des activités quotidiennes}

Une personne handicapée ou dépendante non aidée est donc a priori limitée dans ses activités quotidiennes, à moins de recourir à des dispositifs techniques. Mais la limitation est susceptible de varier

10 Pour de telles descriptions, voir notamment Dutheil 2001 et 2002, Michaudon 2002. 
considérablement selon les activités concernées. C'est pourquoi nous avons distingué des groupes d'activités (cf. tableau 1). II y a des activités qui ne peuvent pas être évitées: manger, boire, se laver, s'habiller, se déplacer etc. Ces activités doivent être effectuées soit sans aide, soit grâce à une aide humaine, faute de quoi les conditions d'une vie ordinaire ne sont plus remplies, comme c'est le cas pour les individus qui ont recours à des aides techniques lourdes (perfusion par exemple) ou qui sont confinés à un espace restreint. Nous proposons de les appeler «vitales». En revanche, des activités comme «entendre une conversation» ou «se souvenir du moment de la journée» ne sont pas indispensables pour mener une vie ordinaire, dans la mesure où I'on peut vivre sans les accomplir, même si I'on mène alors une vie sociale restreinte au regard des normes de notre société. Nous proposons de les appeler «sociales». Enfin, il existe d'autres activités, comme «préparer ses repas» ou «se couper les ongles des orteils» dont on peut se passer en faisant appel à un service professionnel auquel n'importe qui peut avoir accès sous réserve d'être solvable (restaurant, pédicure) ; il est donc possible de ne jamais les effectuer seul sans pour autant avoir recours à des aides dédiées aux personnes handicapées ou dépendantes. Nous proposons de les appeler «substituables».

Tableau 1. Classement des activités retenues

\begin{tabular}{|c|c|c|}
\hline Activités vitales & Activités sociales & Activités substituables \\
\hline $\begin{array}{l}\text { - Faire sa toilette } \\
\text { - S'habiller } \\
\text { - Se servir à boire } \\
\text { - Manger et boire quand c'est prêt } \\
\text { - Aller aux toilettes } \\
\text { - Contrôler ses selles et urines } \\
\text { - Sortir de sa chambre } \\
\text { - Se lever, se coucher } \\
\text { - S'asseoir, se lever } \\
\text { - Se déplacer sur un étage } \\
\text { - Se service de ses doigts, } \\
\text { de ses mains }\end{array}$ & $\begin{array}{l}\text { - Sortir de son domicile } \\
\text { - Descendre, monter un escalier } \\
\text { - Prendre les transports en commun } \\
\text { - Communiquer avec son entourage } \\
\text { - Se souvenir du moment de la journée } \\
\text { - Trouver son chemin } \\
\text { - Voir de près } \\
\text { - Reconnaître un visage } \\
\text { à quatre mètres } \\
\text { - Entendre une conversation } \\
\text { - Parler normalement } \\
\text { - Se pencher, ramasser un objet }\end{array}$ & $\begin{array}{l}\text { - Couper sa nourriture } \\
\text { - Faire ses achats } \\
\text { - Préparer ses repas } \\
\text { - Accomplir les tâches } \\
\text { ménagères } \\
\text { - Remplir un formulaire } \\
\text { simple } \\
\text { - Se couper les ongles } \\
\text { des orteils }\end{array}$ \\
\hline
\end{tabular}

En croisant ces activités et les aides déclarées, nous avons construit un indicateur de niveau d'aide (cf. tableau 2, p. 94). Nous avons hiérarchisé les situations en considérant d'abord que si la personne a recours à une aide technique lourde pour une activité vitale, cette personne est confinée à domicile. En outre, dès lors qu'une aide humaine existe pour une activité vitale, elle a également été qualifiée de vitale. Ensuite, si des aides sociales et des aides substituables sont apportées, nous n'avons 
retenu que I'aide sociale. Si enfin aucune aide humaine ou technique lourde n'est mentionnée, nous avons distingué les quatre situations suivantes: vie sociale restreinte (au moins une activité sociale n'est pas effectuée), vie sans aide avec de grandes difficultés (au moins une activité est effectuée seul mais avec de grandes difficultés), vie sans aide avec quelques difficultés (aucune activité n'est effectuée avec de grandes difficultés, mais une au moins I'est avec quelques difficultés), enfin vie sans aide et sans difficultés.

Tableau 2. Consctruction du niveau d'aide

\begin{tabular}{|c|c|c|c|}
\hline Activités & $\begin{array}{c}\text { Activité } \\
\text { non effectuée }\end{array}$ & $\begin{array}{l}\text { Activité effectuée } \\
\text { avec une aide } \\
\text { humaine }\end{array}$ & $\begin{array}{l}\text { Activité effectuée } \\
\text { sans aide }\end{array}$ \\
\hline Vitales & Confiné à domicile & Aide vitale & \multirow[b]{3}{*}{$\begin{array}{c}\text { Pas d'aide, } \\
\text { avec ou sans } \\
\text { (grandes) difficultés }\end{array}$} \\
\hline Sociales & Vie sociale restreinte & Aide sociale & \\
\hline Substituables & $\begin{array}{c}\text { Pas d'aide, } \\
\text { éventuellement au prix } \\
\text { de difficultés } \\
\text { (grâce à un recours au } \\
\text { marché) }\end{array}$ & Aide substituable & \\
\hline
\end{tabular}

Cet indicateur diffère fortement des indicateurs usuels, comme I'indice de Katz ou celui de Lawton. II n'a pas vocation à déterminer un degré de handicap ou de dépendance bien qu'il soit partiellement hiérarchisé (par exemple, I'aide humaine vitale se rapporte aux personnes qui bénéficient d'une aide humaine pour au moins une activité vitale; I'aide humaine sociale concerne les personnes qui bénéficient d'une aide humaine pour au moins une activité sociale mais n'en ont besoin pour aucune activité vitale; I'aide humaine substituable touche les personnes qui ne bénéficient d'une aide humaine que pour une ou plusieurs activités substituables). La construction de cet indicateur repose sur les types d'arrangements pratiques qu'il est possible de mettre en place pour rester à domicile sans effectuer seul tel genre d'activités (recourir au marché, restreindre sa vie sociale, faire appel à une aide humaine) ; il est en cela mieux adapté à notre objet. II est également mieux adapté à la population que nous avons sélectionnée, qui a pu rester à domicile malgré des difficultés quotidiennes importantes. II permet donc de distinguer différentes formes d'autonomie ou d'arrangements pratiques tandis que les indicateurs usuels nous auraient conduits à considérer la majeure partie de notre population comme autonome, sans plus de précisions. 


\section{Graphique 1. Modalités de niveau d'aide}

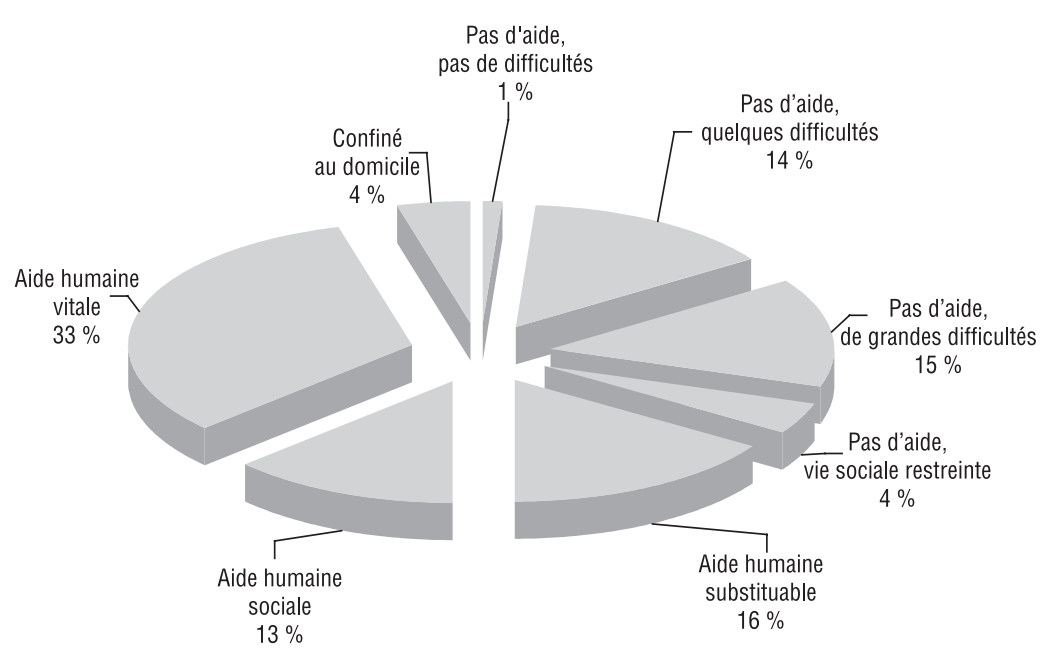

II est frappant de constater, cependant, que le tiers des individus observés effectuent sans aide humaine l'ensemble des activités quotidiennes considérées, parfois au prix d'importantes difficultés (15\% de l'ensemble de notre population), parfois encore au prix d'une vie sociale restreinte (4\%) (cf. graphique 1). Lorsqu'une aide humaine est mentionnée, c'est souvent pour au moins une activité vitale (un tiers de l'ensemble de notre population), mais aussi assez souvent pour des activités uniquement substituables (16\%).

\section{L'insaisissable besoin d'aide}

Les personnes qui ne déclarent aucune aide humaine, et plus encore celles qui déclarent des difficultés à faire face aux activités quotidiennes, sont généralement considérées dans la littérature comme présentant un besoin d'aide non satisfait (Davin et al., 2005). C'est également I'optique des politiques sociales, dans une logique de mise en place de dispositifs d'aide et d'évaluation du nombre de bénéficiaires concernés (Bontout et al., 2002). Nous préférons, pour notre part, éviter d'employer cette notion. Tout d'abord, elle néglige la fierté que peuvent ressentir et exprimer certaines personnes handicapées ou dépendantes à se passer d'aide, malgré les difficultés qu'elles rencontrent. Ensuite, elle suppose que la capacité à être autonome découle plus ou moins mécaniquement du niveau d'incapacité fonctionnelle. Or, ce qu'un individu peut faire sans aide n'est pas indépendant de l'aide qu'il a reçue jusque-là. 
Retraite et Société / Nº 53 Janvier 2008

Ainsi, une personne handicapée secondée par ses proches dans toutes les activités de la vie quotidienne n'a jamais été en situation d'apprendre à effectuer sans aide ces activités, tandis qu'une personne atteinte de la même déficience mais moins encadrée par son entourage aura pu développer des manières de faire (nous les appelons «routines») qui lui permettent d'atteindre, sans aide humaine, une autonomie supérieure. À l'inverse, certaines aides humaines (par exemple un accompagnement pour rendre autonome dans les transports un enfant handicapé mental) favorisent une autonomie ultérieure. Autrement dit, recevoir une aide peut aussi bien enfermer dans une situation d'aide et donc dans une incapacité à se débrouiller seul, que conduire à une autonomie croissante $^{11}$.

Sortir de la logique du besoin d'aide, c'est aussi abandonner une conception normative, qui vise à dicter ce que devraient faire les personnes confrontées à des difficultés dans la vie quotidienne pour mener une vie «normale», au profit d'une optique descriptive, attentive aux arrangements pratiques qui permettent à ces personnes de vivre malgré leurs limitations. La manière dont les questions sur les activités quotidiennes sont formulées dans l'enquête HID va d'ailleurs dans ce sens puisqu'on ne demande pas aux personnes interrogées si elles pourraient faire telle ou telle activité $\operatorname{seules}^{12}$, mais si elles font ces activités et éventuellement comment (avec ou sans I'aide de quelqu'un, avec ou sans difficultés, etc.). II s'agit donc de renoncer à un «misérabilisme» (Grignon, Passeron, 1989) qui perçoit les activités des personnes handicapées ou dépendantes en termes de manques, de besoin d'aide, pour épouser un point de vue sociologique qui décrit comment ces personnes s'arrangent. Mais il faut alors éviter le piège du «populisme» (Grignon, Passeron, 1989) qui consisterait à dire que ces personnes se tirent très bien seules d'affaire et n'ont donc besoin d'aucune aide.

11 Cet aspect est également évoqué par Colin, Robine, 2003 : «En cas de difficultés, l'assistance apportée par une tierce personne pourrait renforcer le sentiment d'un besoin d'aide, et donc de dépendance à l'égard d'un aidant» (p. 9).

12 II reste néanmoins quelques exceptions: se pencher pour ramasser un objet, porter un colis, monter et descendre un escalier, notamment, formulées en termes de capacités (pouvez-vous...? ?) et non d'activités (faites-vous...? ?). On trouve ici trace des hésitations entre une mesure des capacités de la personne et une mesure de ses performances effectives (Cambois, Robine, 2003a). 
LA VIE À DOMICILE DES PERSONNES HANDICAPÉES OU DÉPENDANTES

D'une certaine façon, cette optique revient à enrichir la classification de Philip Wood qui a inspiré la Classification Internationale des Handicaps sur laquelle s'appuie le questionnaire de I'enquête $\mathrm{HID}^{13}$ : le handicap n'est pas réductible à une seule dimension. II ne peut être appréhendé qu'en distinguant le plan des déficiences ${ }^{14}$ (niveau lésionnel), celui des incapacités ${ }^{15}$ (niveau fonctionnel) et celui du désavantage ${ }^{16}$ (niveau social) bien qu'ils soient dans la plupart des cas étroitement reliés (Ravaud et al., 2002). Le passage du niveau fonctionnel au niveau social se fait dans ce modèle par la prise en compte de «l'environnement», généralement réduit aux conditions matérielles d'habitat et d'accès à certaines activités (les loisirs notamment). Bien d'autres éléments sont à prendre en compte pour comprendre le passage des incapacités à la situation concrète de handicap, à commencer par les arrangements pratiques passés et présents, comme le montrent les exemples que nous avons développés plus haut. De manière encore plus générale, c'est I'environnement domestique tout entier qui peut être mobilisé pour éclairer les situations observées.

\section{- De l'aide aux aidants}

La déclaration d'une aide ne doit pas être confondue avec la désignation d'aidants. Beaucoup de personnes ont ainsi déclaré être aidées pour effectuer telle activité, sans pour autant mentionner un aidant Iorsque la question leur était directement posée ${ }^{17}$. C'est sur ce décalage que nous allons à présent nous pencher pour progresser dans la compréhension des arrangements pratiques.

13 La Classification internationale des handicaps $(\mathrm{ClH})$ a été adoptée par l'OMS en 1980 et en France en 1988. Elle a depuis été remplacée par la Classification internationale du fonctionnement, du handicap et de la santé (OMS, 2001). Pour une synthèse sur la $\mathrm{CIH}$ et son évolution, voir Cambois, Robine, 2003a.

14 "Toute perte de substance ou altération d'une structure ou fonction psychologique, physiologique ou anatomique» (OMS, 1980).

15 "Toute réduction (résultant d'une déficience) partielle ou totale, de la capacité d'accomplir une activité d'une façon ou dans des limites considérées comme normales pour un être humain" (OMS, 1980).

16 "II résulte pour un individu donné d'une déficience ou d'une incapacité qui limite ou interdit l'accomplissement d'un rôle normal (en rapport avec l'âge, le sexe, les facteurs sociaux et culturels)» (OMS, 1980).

17 La déclaration d'aidant ne figure pas dans la même partie du questionnaire HID que la description des activités. 


\section{La cohabitation comme condition du maintien à domicile}

Le niveau d'aide est étroitement corrélé à la structure du ménage et les situations les plus critiques sont concentrées dans certains types de ménages. Ainsi, les cas où la personne enquêtée vit chez ses enfants (recohabitation) ou chez ses parents (enfant dans un ménage) sont également ceux où l'aide vitale et le confinement au domicile sont les plus fréquents (cf. graphique 2) : ils représentent respectivement près des trois quarts et près des deux tiers des cas. On observe le même phénomène, à un moindre degré, dans les ménages complexes (la moitié des cas), alors que dans les autres structures de ménages, ces deux modalités de niveau d'aide représentent au maximum le tiers des cas.

\section{Graphique 2. Niveau d'aide et structure du ménage}

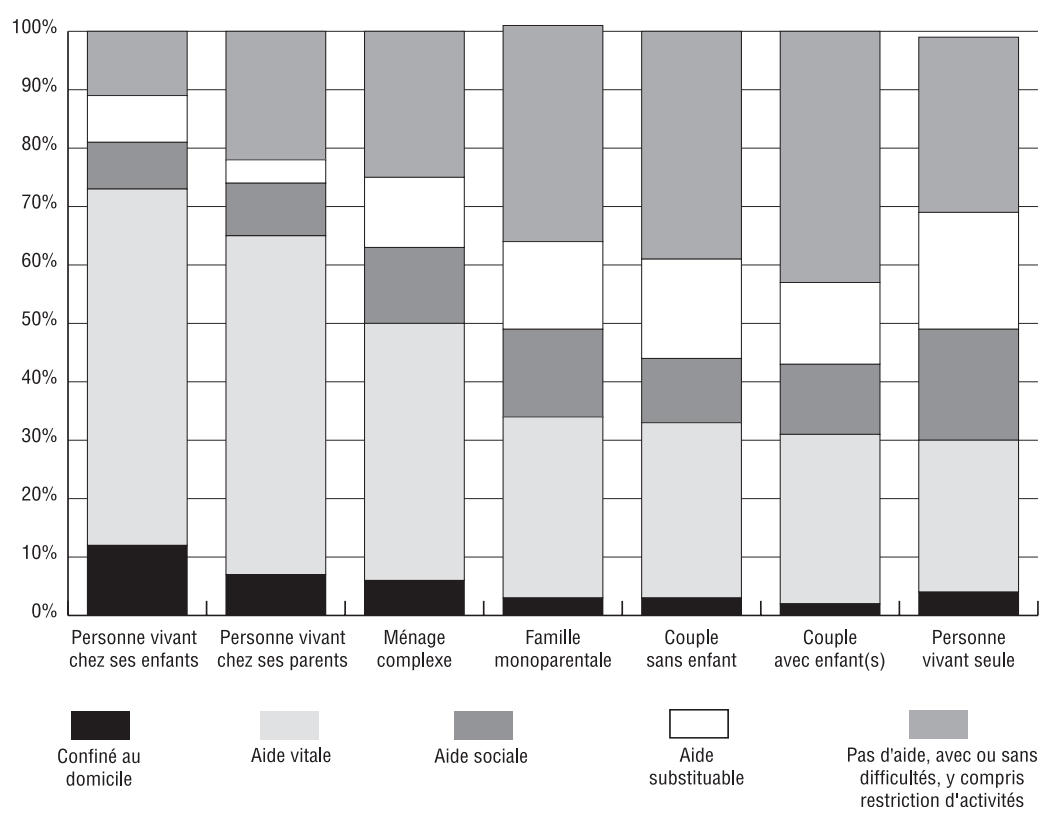

Ces structures de ménage sont également celles où les personnes déclarent le plus souvent des aidants familiaux: un aidant familial (seul ou épaulé par un aidant professionnel) est mentionné dans $81 \%$ des cas de recohabitation et dans $70 \%$ des cas où il s'agit d'un enfant vivant chez ses parents (cf. graphique 3). Dans ces situations, il est très vraisemblable que la cohabitation avec des proches soit la seule alternative à un placement en institution. 
Inversement, les personnes vivant seules sont très rarement en position de confinement à domicile et sont les moins nombreuses à déclarer une aide pour des activités vitales. Mais elles sont les plus nombreuses à déclarer une aide pour des activités sociales ou substituables (cf. graphique 2). On peut donc considérer que si elles parviennent à vivre seules à leur domicile, c'est qu'elles peuvent surmonter leurs difficultés quotidiennes, parfois grâce à une aide humaine: ce sont elles qui ont le plus souvent recours à une aide professionnelle exclusive (cf. graphique 3).

\section{Graphique 3. Statut des aidants selon le type de ménage}

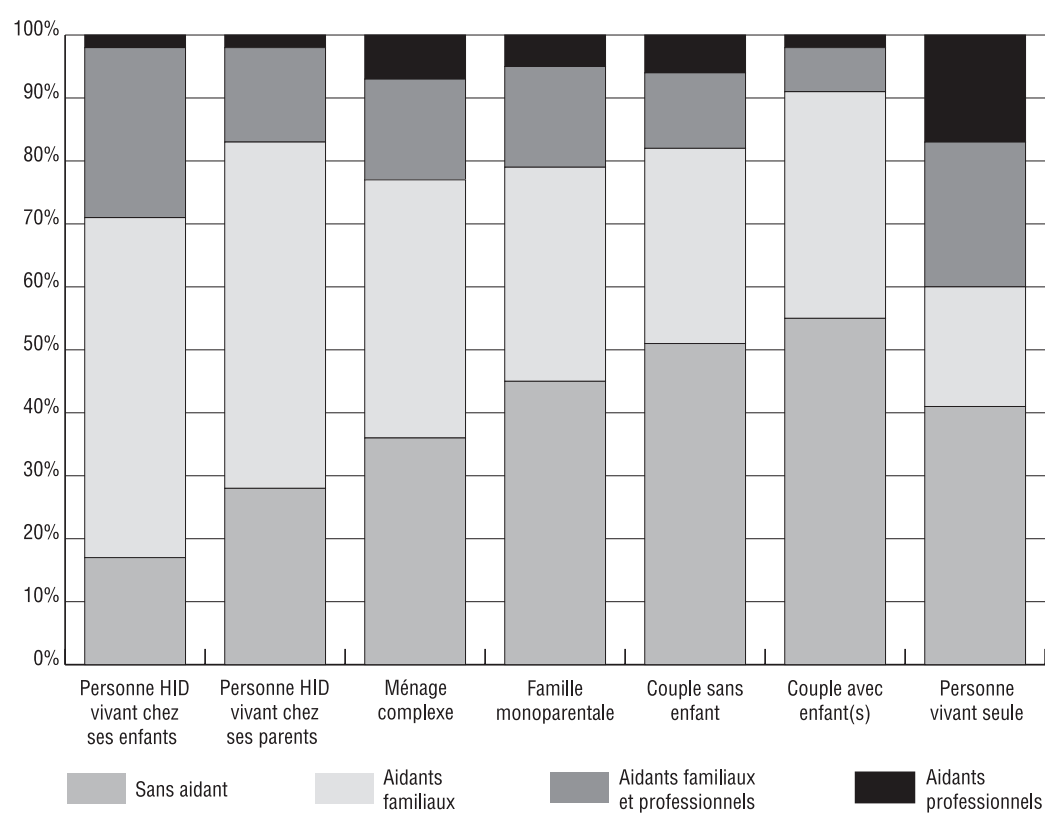

\section{La cohabitation, une modalité d'aide}

Les personnes qui vivent en couple, avec ou sans enfant cohabitant, ou dans une moindre mesure en famille monoparentale, sont les plus nombreuses à ne déclarer aucun aidant (cf. graphique 3). Il est également vrai que, si ces personnes ont un conjoint, et éventuellement un enfant, c'est que leur handicap ne les empêche pas de mener une vie familiale. Elles sont d'ailleurs surreprésentées parmi les plus faibles niveaux d'aide (cf. graphique 2). 
Retraite et Société / Nº 53 Janvier 2008

Mais si la vie en couple est moins souvent associée à la désignation d'un aidant, c'est aussi parce que la participation des membres du ménage à I'organisation quotidienne, en particulier celle du conjoint, n'est pas toujours perçue et désignée comme de l'aide, même quand cette participation augmente avec l'apparition et l'aggravation des incapacités. Les personnes en couple ne déclarant aucun aidant vivent effectivement une forme d'autonomie, mais cette autonomie est bien domestique et non individuelle.

Cela ne signifie pas que I'aide offerte par les membres du ménage est systématiquement sous-déclarée. Mais on peut faire I'hypothèse que la réorganisation des tâches domestiques n'aboutit à la désignation d'un ou plusieurs aidants que lorsqu'elle est suffisamment forte. Ainsi, dans les cas de recohabitation avec un parent âgé, ou de maintien à domicile d'un enfant handicapé, c'est bien le bouleversement de l'organisation domestique qui rend I'aide visible et les aidants désignables en tant que tels. Seules les aides extraordinaires sont qualifiées comme des aides, et ceux qui les prodiguent comme des aidants, au contraire de la participation aux tâches domestiques considérée comme normale. En ce sens, cohabiter, c'est déjà aider, mais la désignation d'aidants souligne un sens particulier donné à la relation. Le seuil à partir duquel la participation domestique devient de l'aide s'inscrit donc dans le contexte des relations au sein du ménage et varie selon diverses caractéristiques sociales, à commencer par le genre: I'implication domestique des hommes sera bien plus rapidement vue comme une aide à leur conjointe défaillante que I'inverse ${ }^{18}$.

\section{Aidants familiaux, aidants professionnels}

Si I'aide prodiguée par un cohabitant de la famille n'est perçue comme telle que dans les cas «extraordinaires», on peut en dire autant de I'aide apportée par certains professionnels. La perte d'indépendance liée au vieillissement est par exemple ressentie de manière tout à fait différente selon que I'on avait ou non recours à des services rémunérés (emploi de personnel domestique, visites régulières chez le pédicure, etc.) avant que les incapacités apparaissent. L'utilisation de ce type de services est déclarée par les personnes concernées comme une aide uniquement quand elle est motivée ou renforcée par l'apparition d'incapacités. Presque la moitié (46\%) des personnes aidées pour des activités substituables ne sont d'ailleurs aidées par personne (cf. graphique 4). aidées que les hommes (Ravaud, Ville, 2003). 
LA VIE À DOMICILE DES PERSONNES HANDICAPÉES OU DÉPENDANTES

C'est bien I'indice que I'aide substituable, qu'elle soit fournie gratuitement par un membre du ménage ou par des professionnels rémunérés ne relevant pas du domaine sanitaire, n'est pas nécessairement perçue comme une réponse à d'éventuels handicaps ou problèmes de santé ${ }^{19}$. Le même phénomène de sous-déclaration des aidants s'observe, à une moindre échelle, pour I'aide à des activités sociales (23\% de ceux qui déclarent une aide sociale ne désignent pas d'aidant). La sous-déclaration des aidants disparaît en revanche lorsqu'il s'agit d'aide pour des activités vitales: les personnes qui interviennent pour ces activités sont bel et bien signalées comme des «aidants».

\section{Graphique 4. Niveau d'aide et statut des aidants}

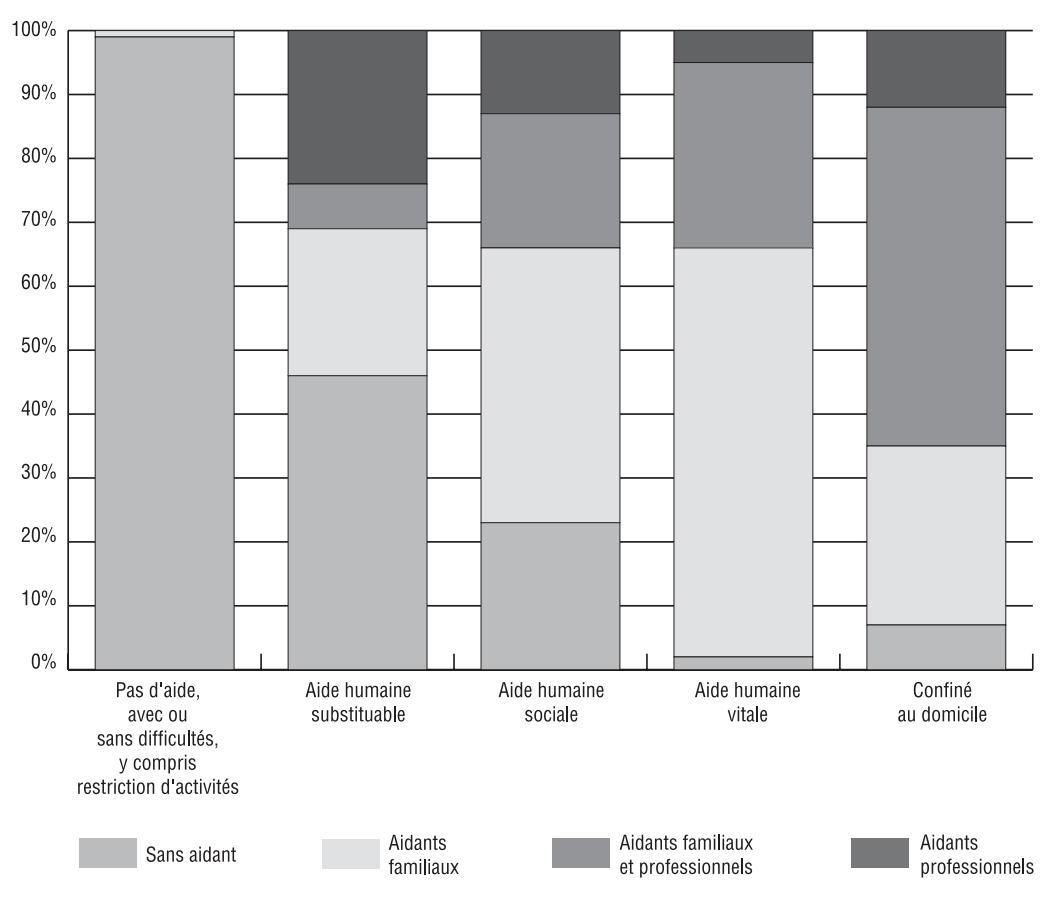

Lorsque des aidants sont déclarés pour une aide substituable, il s'agit à parts égales (dans près d'un quart des cas à chaque fois) d'aidants exclusivement familiaux ou professionnels et presque jamais d'une coopération entre aidants familiaux et professionnels (cf. graphique 4).

19 C'est dans ces termes qu'est formulé le questionnaire de l'enquête. Une personne qui emploie une femme de ménage, mais qui ne peut le motiver par un problème de santé ou un handicap, n'est pas censée mentionner son employée de maison comme aidante. 
Retraite et Société / Nº 53 Janvier 2008

L'aide sociale est plutôt assurée par des aidants familiaux seulement (à $43 \%$ ), mais de façon non négligeable par des aidants professionnels (seuls ou en collaboration avec la famille). Enfin, I'aide vitale est fournie aux deux tiers par des aidants familiaux et très rarement par des aidants uniquement professionnels. Ce n'est donc qu'en dernière extrémité que les professionnels interviennent massivement, mais principalement aux côtés de la famille: 53 \% des personnes que nous qualifions de confinées au domicile déclarent une coopération entre aide professionnelle et familiale, $27 \%$ une aide exclusivement familiale et $12 \%$ une aide exclusivement professionnelle.

\section{- Les principales formes d'arrangements pratiques}

Afin d'aller plus loin dans la description des arrangements pratiques, et de mieux prendre en compte les caractéristiques des individus et des ménages dans lesquels ils vivent, nous avons eu recours à une analyse des correspondances multiples (ACM). Le nuage de points du graphique 5 en donne la représentation sur les deux premiers axes ${ }^{20}$.

Alors que le premier axe (horizontal) oppose notamment les personnes ne déclarant aucun aidant à celles qui bénéficient d'une aide strictement familiale, le second (vertical) oppose plutôt celles qui ne déclarent aucun aidant à celles qui bénéficient d'une aide exclusivement ou partiellement professionnelle. Quatre pôles apparaissent, qui permettent d'articuler la position dans le cycle de vie, les difficultés rencontrées et l'existence d'une aide, ainsi que sa nature si elle existe effectivement.

Le premier de ces quatre pôles (à gauche), que nous proposons de nommer «autonomie domestique ${ }^{21}$ », concerne des individus en couple, sans enfant, dont les arrangements pratiques, sûrement internes au ménage, n'ont pas suffisamment bouleversé l'organisation de la vie quotidienne pour être perçus et désignés comme des aides.

20 Les variables actives sont l'indicateur de niveau d'aide, le statut des aidants, la nature des déficiences, l'âge, l'ancienneté des dernières difficultés, le caractère cumulatif ou non des difficultés, la reconnaissance officielle ou non d'un handicap, le type de ménage et le revenu par unité de consommation de ce ménage.

21 Nous utilisons le terme d'autonomie dans une acception différente de l'usage habituel: il s'agit pour nous de décrire des personnes sans aide déclarée. II faut toutefois remarquer que cet usage n'est pas incompatible avec la définition de l'autonomie comme autonomie de décision individuelle, puisque c'est bien de ce côté du graphique que se projettent les individus n'ayant pas de déficiences intellectuelles. 


\section{Graphique 5. Niveau d'aide et statut des aidants}

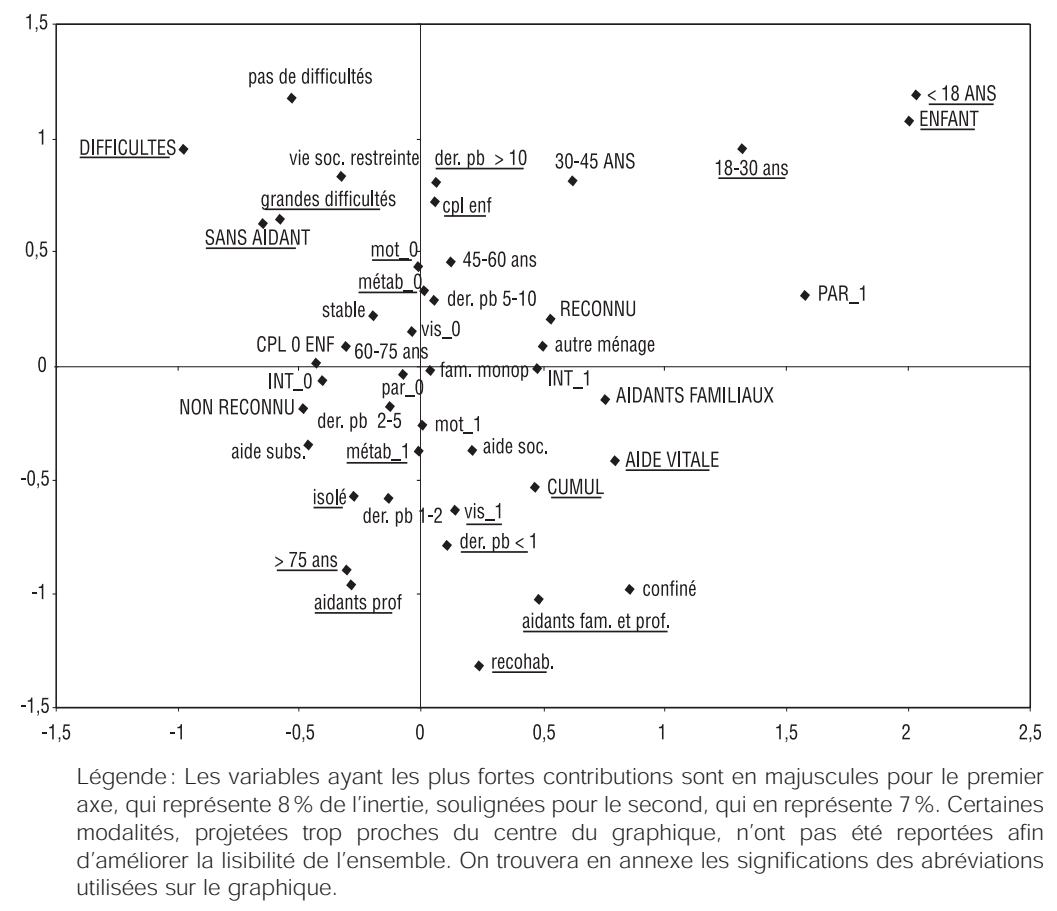

S'ils parviennent à maintenir une autonomie malgré des difficultés parfois importantes, les personnes du second pôle (en haut) se distinguent par I'ancienneté importante de leurs incapacités. Elle a pu favoriser la mise en place de «routines» permettant de se passer d'une aide humaine ou du moins, encore une fois, de ne pas modifier suffisamment I'organisation quotidienne pour que cette organisation soit qualifiée $\mathrm{d}^{\prime} \mathrm{aide}^{22}$. Nous désignons ce deuxième pôle par le terme $\mathrm{d}^{\prime}$ «aménagement ».

Les deux pôles suivants se caractérisent par le statut des aidants et la position dans le cycle de vie. Le troisième (en bas) concerne des personnes âgées de plus de 75 ans ayant recours à une aide pour des activités vitales et faisant appel à des aidants professionnels (éventuellement en association avec des aidants familiaux).

22 Paradoxalement, malgré l'ancienneté importante des incapacités, on trouve plutôt dans ce pôle des personnes de moins de 30 ans. On peut faire l'hypothèse que la mise en place de "routines » permettant de se passer d'aide est plus difficile pour des personnes plus âgées, à partir d'un stade où les habitudes sociales et quotidiennes sont bien ancrées. 
Retraite et Société / N²53 Janvier 2008

On reconnaît là la cible des politiques de la vieillesse, en particulier dans une logique de maintien à domicile des personnes âgées dépendantes (Join-Lambert, 1997 ; Martin 2003; Clément, Lavoie 2005). Nous appelons ce pôle «dépendance», ce mot revêtant ici un sens plus précis que la catégorie politique correspondante dans la mesure où il est fortement associé à une crise (puisque les dernières incapacités sont récentes).

Le dernier pôle (à droite) correspond également à des occurrences où des aidants sont déclarés, mais concerne des individus beaucoup plus jeunes. La reconnaissance officielle d'un handicap étant également très fortement associée à ce pôle, on perçoit cette fois-ci une autre cible des politiques publiques: les personnes handicapées (Blanc, Stiker, 1998). Nous avons, là encore, une acception plus restrictive du terme «handicap» que celle en vigueur dans les catégories de l'action publique. En effet, le pôle mis en évidence par l'analyse factorielle repose également sur une aide vitale et sur des aides apportées par des membres de la famille, et ne désigne pas l'ensemble des personnes handicapées bénéficiant d'une reconnaissance institutionnelle.

Cette analyse des arrangements pratiques met en évidence qu'une part non négligeable de cette population ne déclare aucune aide humaine. Pour rendre compte de ce résultat, il convient de garder à l'esprit deux remises en question du sens commun quant au problème des personnes handicapées ou dépendantes. Tout d'abord, il ne faut pas sous-estimer la force des arrangements et des «routines» qui permettent à des individus subissant des limitations de les affronter seules. Un constat appuie cette thèse: ce sont les incapacités les plus anciennes qui sont le plus souvent vécues sans aide humaine déclarée. En outre, il ne faut pas opposer trop radicalement les personnes qui déclarent des aidants et celles qui n'en déclarent pas, sans prendre en compte au préalable la structure du ménage.

Par ailleurs, parmi les personnes qui déclarent des aidants, on trouve les deux cibles des politiques sociales du handicap et de la vieillesse: d'un côté, une population jeune, reconnue administrativement, aidée uniquement par la famille, de l'autre des personnes âgées qui disposent d'une aide professionnelle souvent complémentaire de I'aide familiale. L'approche à partir des arrangements pratiques effectivement mis en place au sein des ménages converge avec une description des individus bénéficiant de telle ou telle politique sociale (handicap ou dépendance). Pour autant, on repère entre les pôles que nous dégageons de I'analyse et les cibles des politiques sociales, des décalages révélateurs de certains effets de ces politiques. Ainsi, I'accès à une aide professionnelle hors institution est beaucoup plus difficile pour les handicapés que pour les 
personnes âgées dépendantes (puisque nous ne considérons que la population vivant à domicile). $\mathrm{Si}$, pour les handicapés comme pour les personnes âgées dépendantes, la cohabitation avec des proches (ascendants ou descendants respectivement) est une modalité d'aide, le maintien à domicile avec I'appui exclusif de professionnels, semble spécifique aux personnes âgées. Non seulement les politiques de la vieillesse permettent un recours accru aux aidants professionnels, mais elles s'adressent à différents types de bénéficiaires: d'une part les personnes âgées dépendantes (où les professionnels interviennent comme appui de I'entourage), d'autre part les personnes vieillissantes, parfois isolées (où les professionnels interviennent à la place de I'entourage). Les arrangements pratiques ne sont pas indépendants du contexte institutionnel et politique dans lequel ils s'inscrivent, même s'ils conservent une certaine autonomie.

\section{- Conclusion}

Nos résultats soulignent l'importance de la prise en compte de la structure du ménage dans la construction des arrangements pratiques. La désignation d'aidants est en effet le résultat d'un processus qui caractérise la participation aux tâches quotidiennes comme une aide, une aide rendue visible car perçue comme extraordinaire, soit que I'organisation domestique ait été bouleversée par l'arrivée de nouveaux aidants, éventuellement professionnels, soit qu'elle ait été substantiellement transformée par l'apparition des incapacités ou leur aggravation. Cette visibilité de I'aide varie selon le genre ou encore la structure du ménage de la personne handicapée ou dépendante, mais aussi selon le degré de professionnalisation des activités quotidiennes au sein de la société. Plus ces activités sont pensées comme des activités qui peuvent être accomplies par des professionnels et plus elles sont vécues comme des aides, même accomplies par des proches. C'est parce que I'organisation des tâches domestiques est jusqu'à un certain point pensée comme naturelle que la définition de I'aidant, en particulier à l'intérieur d'un ménage, est floue.

La professionnalisation de ces activités reste inégale. Dans le cas des activités que nous avons appelées substituables, c'est-à-dire qui peuvent être évitées par un recours au marché, le problème principal est celui de la solvabilité, que des politiques de subventions ciblées peuvent suffire à régler. Le recours à des professionnels peut également être envisagé, comme c'est d'ailleurs déjà assez largement le cas. En revanche, dans le cas des activités dites sociales, ce sont plutôt les proches qui sont «naturellement» amenés à aider, parfois au détriment de leur propre vie 
Retraite et Société / Nº 53 Janvier 2008

sociale. Mais c'est encore plus frappant dans le cas des activités vitales, pour lesquelles les aidants familiaux sont très majoritairement mobilisés, cette fois-ci pour une aide extraordinaire qui est donc bien définie comme telle.

Si les conditions pour transformer ces aides extraordinaires en emplois sont relativement bien réunies dans le cadre des politiques de la dépendance, c'est beaucoup moins le cas dans le cadre des politiques du handicap. Nos résultats montrent en effet que le recours à des aidants professionnels est bien plus fréquent pour les personnes âgées rencontrant des incapacités récentes que pour des personnes plus jeunes et reconnues comme handicapées. Les réformes récentes qui ont trouvé leur point d'orgue avec la loi de 2005 se sont notamment donné pour objectif de faire converger ces politiques sectorielles, en réunifiant au mieux handicap et dépendance. Mais il est encore trop tôt pour apprécier les effets de ces nouvelles politiques sociales sur I'organisation quotidienne des personnes handicapées et dépendantes.

Pour finir, rappelons que les résultats présentés ici n'ont de sens que si I'on garde à l'esprit qu'ils ne concernent que la population, socialement sélectionnée, des personnes vivant en domicile ordinaire alors même qu'elles rencontrent des obstacles importants dans les activités quotidiennes $^{23}$. Les effets de transition vers des institutions, qui correspondent donc à une sortie de notre échantillon, n'ont pu être observés ici, de même que les autres transitions qui rendent fluides et continus les arrangements pratiques analysés ici de manière statique ${ }^{24}$.

23 Les variables de position et d'origine sociale n'interviennent pas de manière significative dans notre analyse parce que leurs effets s'exercent en amont, sur la sélection entre domicile et institution (Désesquelles, Brouard, 2003; Mormiche, Boissonnat, 2003); il en va de même des effets de genre (Dufour-Kippelen, 2000).

24 Sur l'importance de ces transitions, on pourra se reporter à l'article d'Émilie Biland dans ce même numéro p. 68. 


\section{- Annexe}

Variables utilisées dans les analyses factorielles et légendes des graphiques

\begin{tabular}{|c|c|c|}
\hline Variables et modalités & $\begin{array}{l}\text { Abréviations sur les } \\
\text { graphiques }\end{array}$ & Fréquences \\
\hline $\begin{array}{l}\text { Niveau d'aide } \\
\text { Confiné à domicile } \\
\text { Aide vitale } \\
\text { Aide sociale } \\
\text { Aide substituable } \\
\text { Évitement de certaines activités } \\
\text { Pas d'aide, de grosses difficultés } \\
\text { Pas d'aide, des difficultés } \\
\text { Pas d'aide, pas de difficulté }\end{array}$ & $\begin{array}{c}\text { confiné } \\
\text { aide vitale } \\
\text { aide soc. } \\
\text { aide subs. } \\
\text { vie soc. restreinte } \\
\text { grandes difficultés } \\
\text { difficultés } \\
\text { pas de difficulté }\end{array}$ & $\begin{array}{c}4 \\
33 \\
13 \\
13 \\
16 \\
4 \\
15 \\
14 \\
1\end{array}$ \\
\hline $\begin{array}{l}\text { Déficiences } \\
\text { Déficience motrice } \\
\text { Pas de déficience motrice } \\
\text { Déficience visuelle } \\
\text { Pas de déficience visuelle } \\
\text { Déficience auditive } \\
\text { Pas de déficience auditive } \\
\text { Déficience de la parole } \\
\text { Pas de déficience de la parole } \\
\text { Déficience métabolique } \\
\text { Pas de déficience métabolique } \\
\text { Déficience mentale } \\
\text { Pas de déficience mentale }\end{array}$ & $\begin{array}{l}\text { mot_1 } \\
\text { mot_0 } \\
\text { vis_1 } \\
\text { vis_0 } \\
\text { aud_1 } \\
\text { aud_0 } \\
\text { par_1 } \\
\text { par_0 } \\
\text { dig_1 } \\
\text { dig_0 } \\
\text { int_1 } \\
\text { int_0 }\end{array}$ & $\begin{array}{l}56 \\
44 \\
18 \\
82 \\
33 \\
67 \\
4 \\
96 \\
31 \\
69 \\
25 \\
75\end{array}$ \\
\hline $\begin{array}{l}\text { Ancienneté de la dernière incapacité } \\
\text { Moins d'un an } \\
\text { de } 1 \text { à } 2 \text { ans } \\
\text { de } 2 \text { à } 5 \text { ans } \\
\text { de } 5 \text { à } 10 \text { ans } \\
\text { Plus de } 10 \text { ans }\end{array}$ & $\begin{array}{c}\text { der. } \mathrm{pb}<1 \\
\text { der. } \mathrm{pb} 1-2 \\
\text { der. pb } 2-5 \\
\text { der. } \mathrm{pb} 5-10 \\
\text { der. } \mathrm{pb}>10\end{array}$ & $\begin{array}{l}19 \\
13 \\
24 \\
19 \\
25\end{array}$ \\
\hline $\begin{array}{l}\text { Reconnaissance administrative } \\
\text { Reconnu } \\
\text { Non reconnu }\end{array}$ & $\begin{array}{c}\text { reconnu } \\
\text { non reconnu }\end{array}$ & $\begin{array}{l}46 \\
54\end{array}$ \\
\hline $\begin{array}{l}\text { Statut des aidants } \\
\text { Sans aidant } \\
\text { Aidé par famille uniquement } \\
\text { Aidé par famille et professionnels } \\
\text { Aidé par professionnels uniquement }\end{array}$ & $\begin{array}{l}\text { sans aidant } \\
\text { aidants familiaux } \\
\text { aidants fam. et prof. } \\
\text { aidants prof. }\end{array}$ & $\begin{array}{c}45 \\
32 \\
15 \\
8\end{array}$ \\
\hline
\end{tabular}


Variables utilisées dans les analyses factorielles et légendes des graphiques (suite)

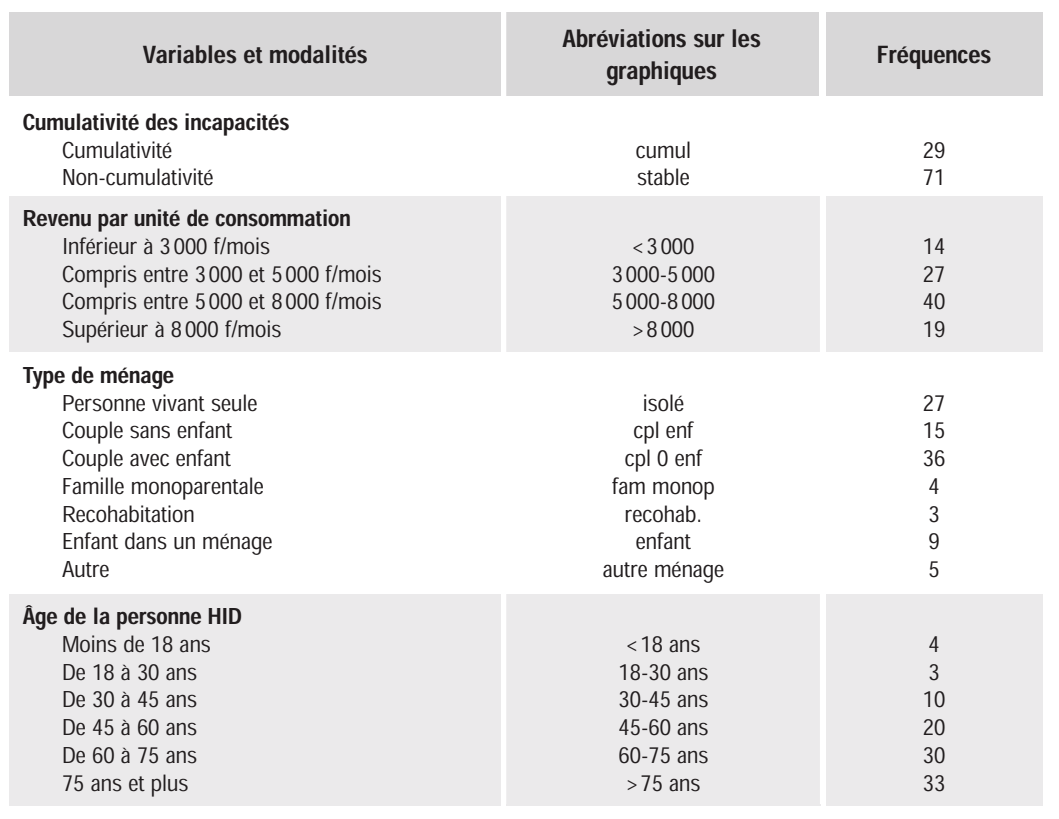




\section{- Bibliographie}

Azéma B., Martinez N., 2005, «Les personnes handicapées vieillissantes: espérance de vie et de santé, qualité de vie. Une revue de la littérature», Revue Française des Affaires Sociales, $\mathrm{n}^{\circ} 2$, p. 297-334.

Barral C., Chauvière M., Paterson F., Stiker H.-J. (dir.), 2000,

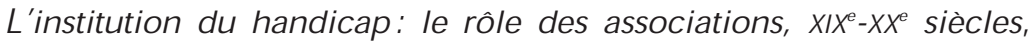
Presses universitaires de Rennes, $320 \mathrm{p}$.

BAUER D., 2007, «Entre maison, enfant(s) et travail : les diverses formes d'arrangement dans les couples», Drees, Études et Résultats, $n^{\circ} 570$, $8 \mathrm{p}$.

Bessière C., 2004, «Les "arrangements de famille". Équité et transmission d'une exploitation familiale viticole», Sociétés contemporaines, $\mathrm{n}^{\circ} 56$, p. 69-89.

Biland É., Eideliman J.-S., Gojard S., Weber F. (dir.), 2004, Dispositifs institutionnels et soutien familial. Pour comprendre les inégalités sociales dans la prise en charge du handicap, Rapport de recherche pour la MiRe.

Blanc A., StikeR H.-J. (dir.), 1998, L'insertion professionnelle des personnes handicapées en France, Paris, Desclée de Brouwer.

Bontout O., Colin C., Kerjosse R., 2002, «Personnes âgées dépendantes et aidants potentiels: une projection à I'horizon 2040 », Drees, Etudes et Résultats, n 160, 12 p.

BresSÉ S., Dutheil N., 2003, «Les bénéficiaires des services d'aide à domicile», Dossiers Solidarité et santé, n 1, p. 17-24.

Cambois E., Robine J.-M., 2003a, «Concepts et mesure de I'incapacité : définitions et application d'un modèle à la population française», Cnav, Retraite et Société, La Documentation française, $\mathrm{n}^{\circ} 39$, p. 60-91.

Cambois E., Robine J.-M., 2003b, «Vieillissement et restriction d'activité: I'enjeu de la compensation des problèmes fonctionnels», Drees, Études et Résultats, n 261, 12 p.

Clément S., Lavole J.-P. (dir.), 2005, Prendre soin d'un proche âgé. Les enseignements de la France et du Québec, Éres, 286 p.

Colın C., 2003, «Que nous apprend I'enquête HID sur les personnes âgées dépendantes, aujourd'hui et demain?», Revue française des Affaires sociales, $\mathrm{n}^{\circ} 1-2$, p. 77-102. 
Davin B., Paraponaris A., Verger P., 2005, « Facteurs démographiques et socio-économiques associés aux besoins d'aide des personnes âgées vivant à domicile: une étude à partir de l'enquête HandicapsIncapacités-Dépendance», Revue d'Epidémiologie et de Santé Publique, n 53, p. 509-524.

Debordeaux D., Strobel P. (dir.), 2002, Les Solidarités familiales en questions. Entraide et transmission, Paris, LGDJ, p. 19-35.

Désesquelles A., Brouard N., 2003, «Le réseau familial des personnes âgées de 60 ans ou plus vivant à domicile ou en institution», Population, n 58, p. 201-228.

Didier-Courbin P., Gilbert P., 2005, «Éléments d'information sur la législation en faveur des personnes handicapées en France: de la loi de 1975 à celle de $2005 »$, Revue française des Affaires sociales, $\mathrm{n}^{\circ} 2$, p. 209-228.

DUfOUR-KIPPELEN S., 2000, Trajectoires de santé des personnes âgées de 60 ans et plus hébergées en institution, Rapport de recherche pour la MiRe.

DutheIL N., 2002, «Les aidants des adultes handicapés», Drees, Études et Résultats, $\mathrm{n}^{\circ} 186,8 \mathrm{p}$.

DutHEIL N., 2001, «Les aides et les aidants des personnes âgées», Drees, Études et Résultats, n 142, 12 p.

Ehrenberg A., 1998, La Fatigue d'être soi. Dépression et société, Odile Jacob, $410 \mathrm{p}$.

ESPAGNOL P., 2007, "L'allocation personnalisée d'autonomie au 31 décembre 2006 », Drees, Études et Résultats, n 569, 4 p.

FRINAULT T., 2005, «La dépendance ou la consécration française d'une approche ségrégative du handicap », Politix, n 72, p. 11-3.

Grignon C., PASSeRon J.-C., 1989, Le Savant et le populaire. Misérabilisme et populisme en sociologie et en littérature, Le Seuil, Paris, $260 \mathrm{p}$.

JoËL M.-Ė., 2002, «Organiser des soins en réseau», in Baszanger I., Bungener M., Paillet A. (dir.), Quelle médecine voulons-nous?, La Dispute.

Join-Lambert M.-T. (dir.), 1997, Politiques sociales, Presses de la FNSP.

KERJOSSE R., 2002, «L'allocation personnalisée d'autonomie au 31 mars $2002 »$, Drees, Études et Résultats, n 178.

110 Laugier S., Paperman P. (dir.), 2006, Éthique et politique du care, éditions de I'EHESS. 
MARTIN C., 2002, «Les solidarités familiales: bon ou mauvais objet sociologique?», in Debordeaux D., Strobel P. (dir.), Les Solidarités familiales en questions. Entraide et transmission, LGDJ, p. 41-72.

Martin C. (dir.), 2003, La dépendance des personnes âgées. Quelles politiques en Europe?, Presses universitaires de Rennes et éditions de I'ENSP.

Michaudon H., 2002, «Les personnes handicapées vieillissantes: une approche à partir de I'enquête HID», Drees, Études et Résultats, $\mathrm{n}^{\circ} 204,12 \mathrm{p}$.

Mormiche P., Boissonnat V., 2003, «Handicap et inégalités sociales: premiers apports de l'enquête "Handicaps, incapacités, dépendance" », Revue française des Affaires sociales, $n^{\circ}$ 1-2, p. 267-285.

MoRmiche P., 2003, «L'enquête "Handicaps, incapacités, dépendance": apports et limites», Revue française des Affaires sociales, $\mathrm{n}^{\circ} 1-2$, p. 13-29.

Oms, 1980, Classification Internationale des Handicaps, 126 p.

Oмs, 2001, Classification Internationale du Fonctionnement, du Handicap et de la Santé, 307 p.

Pennec S., 2005, «Entre règles publiques et arrangements privés: le travail filial et la préservation des biens de famille», Enfances, Familles, Générations, n² 2.

RaVAud J.-F., VitLE I., 2003, «Les disparités de genre dans le repérage et la prise en charge des situations de handicap », Revue française des Affaires sociales, $\mathrm{n}^{\circ}$ 1-2, p 227-253.

Ravaud J.-F., Letourmy A., Ville I., 2002, «Les Méthodes de délimitation de la population handicapée: I'approche de I'enquête de I'Insee Vie quotidienne et santé», Population, n 57, p. 541-566.

ROMien P., 2005, «À I'origine de la réinsertion professionnelle des personnes handicapées: la prise en charge des invalides de guerre», Revue française des Affaires sociales, n² 2, p. 229-248.

ThOMAS H., 2005, «Le "métier" de vieillard. Institutionnalisation de la dépendance et processus de désindividualisation dans la grande vieillesse», Politix, n 72, p. 33-56.

Weber F., Gojard S., Gramain A. (dir.), 2003, Charges de famille. Dépendance et parenté dans la France contemporaine, La Découverte, Paris, $420 \mathrm{p}$. 